\title{
Thyroid Cytology: The Japanese System and Experience at Yamashita Thyroid Hospital
}

Shinya Satoh · Hiroyuki Yamashita Kennichi Kakudo ${ }^{1}$

Department of Endocrine Surgery, Yamashita Thyroid Hospital, Fukuoka; 'Department of Pathology, Kindai University Faculty of Medicine, Nara Hospital, lkoma, Japan

Received: August 25, 2017

Revised: September 23, 2017

Accepted: September 29, 2017

Corresponding Author

Shinya Satoh, MD

Department of Endocrine Surgery, Yamashita

Thyroid Hospital, 1-8 Shimogofuku-machi, Fukuoka

City, Fukuoka 812-0034, Japan

Tel: +81-92-281-1300

Fax: +81-92-281-1301

E-mail: shinya.s.48128@kojosen.com
In Japan, fine-needle aspiration (FNA) cytology is the most important diagnostic modality for triaging patients with thyroid nodules. A clinician (endocrinologist, endocrine surgeon, or head and neck surgeon) generally performs FNA cytology at the outpatient clinic, and ultrasound (US)-guided FNA is widespread because US is extremely common and most clinicians are familiar with it. Although almost all FNA thyroid samples are examined by certified cytopathologists and pathologists, some clinicians assess cytological specimens themselves. In Japan, there are two clinical guidelines regarding the management of thyroid nodules. One is the General Rules for the Description of Thyroid Cancer (GRDTC) published by the Japanese Society of Thyroid Surgery (JSTS) in 2005, and the other is the national reporting system for thyroid FNA cytology published by the Japan Thyroid Association in 2013 (Japanese system). Although the Bethesda System for Reporting Thyroid Cytopathology (Bethesda system) is rarely used in Japan, both the GRDTC and Japanese system tried to incorporate the Bethesda system so that the cytological diagnoses would be compatible with each other. The essential point of the Japanese system is stratification of follicular neoplasm (FN) into three subgroups based on cytological features in order to reduce unnecessary diagnostic thyroidectomy, and this system has been successful in stratifying the risk of malignancy in FN patients at several high-volume thyroid surgery centers. In Japan, the measurement of thyroglobulin and/or calcitonin in FNA needle washings is often used as an adjunct for diagnosis of possible cervical lymph node metastasis when FNA cytology is performed.

Key Words: Thyroid; Fine needle aspiration cytology; Indeterminate; The Bethesda System for Reporting Thyroid Cytopathology; Japan; Risk stratification; Risk of malignancy

\section{BRIEF HISTORY OF THYROID FINE-NEEDLE ASPIRATION}

Shortly after Söderström in Sweden described thyroid fineneedle aspiration (FNA) cytology in 1952, it was introduced to Japan. ${ }^{1,2}$ In as early as 1972, Toriya published his first report on thyroid FNA cytology (in Japanese with an English abstract) from Ito Hospital (a thyroid center in Tokyo, Japan) ${ }^{3}$ where more than 1,000 surgical procedures for thyroid disease were carried out every year. His cytological diagnostic criteria were descriptive and similar to those for pathological diagnosis of surgical specimens. In 2005, Toriya ${ }^{4}$ reported his results and cytological-histological correlations in 1,702 surgically treated Japanese patients. He found 68 patients ( $4.0 \%$ of those receiving surgical treatment) in the indeterminate category, including 39 with benign lesions and 29 with malignancies, and the risk of malignancy (ROM) was $42.6 \%$ in the indeterminate category. ${ }^{4}$ In that report, he divided the indeterminate category into two subcategories (favor benign and favor malignant). Among 28 patients in the favor malignant sub-category, 16 had malignancy (4 papillary thyroid carcinomas [PTCs] and 12 follicular thyroid carcinomas [FTCs]) and the ROM was $57.1 \%$. Among 40 patients in the favor benign subcategory, 13 had malignancy (3 PTCs and 10 FTCs) and the ROM was $32.5 \%$. $^{4}$ This principle to sub-classify indeterminate nodules into low-risk and high-risk categories was similar to the Bethesda System, but the actual ROMs obtained by Toriya ${ }^{4}$ in surgically treated nodules were higher than the implied ROMs for the atypia of undetermined significance (AUS)/follicular lesions of uncertain significance (FLUS) $(5 \%-15 \%)$ and follicular neoplasm (FN)/suspicious for follicular neoplasm (15\%-30\%) categories estimated by the Bethesda system textbook. ${ }^{5}$

\section{FINE-NEEDLE ASPIRATION THYROID CYTOLOGY: SAMPLING AND INTERPRETATION}

In Japan, the clinician (endocrinologist, endocrine surgeon, 
or head and neck surgeon) generally performs FNA cytology at an outpatient clinic and FNA sampling by cytopathologists is unusual. Ultrasonography (US) is widely available and inexpensive (it costs only 35 U.S. dollars, about 10 U.S. dollars [30\%] paid by patients and 25 U.S. dollars covered by the Japanese national health insurance system). Thus, US-guided FNA is preferred by most clinicians. Pathologists in Japan usually have dual certification; they are certified for anatomical pathology by the Japanese Society of Pathology and also for cytopathology by the Japanese Society of Clinical Cytology (JSCC). One of the characteristics of Japanese cytology practice is that a significant number of cytopathologists have a second clinical speciality, such as gynecology, endocrine surgery, breast surgery, respiratory medicine, respiratory surgery, urology, dental surgery, etc. They usually make a cytological diagnosis after collecting the specimens themselves. ${ }^{6}$ Approximately half of all thyroid FNA samples are diagnosed at commercial laboratories by board-certified pathologists and the other half are assessed at hospitals by pathologists and doctors who are board-certified cytologists.

\section{CYTOTECHNOLOGIST TRAINING PROGRAM AND JAPANESE SOCIETY OF CLINICAL CYTOLOGY CERTIFICATION}

The JSCC holds examinations for qualification of cytotechnologists and those who pass are registered as JSCC certified cytotechnologists. There are two pathways to obtaining national board recognition as a medical technologist in Japan; candidates must complete either a 3-year course at a vocational school or a 4-year course at a medical technology school, after which graduates can sit the national examination for medical technologists. Those who obtain a national medical technology license can apply to sit the JSCC examination for cytotechnologists after working for one year at the cytology laboratory of a teaching hospital. More than 800 teaching hospitals in Japan provide education on cytological diagnosis and technical skills, along with cytology laboratory experience. There are also college level (4-year) schools whose graduates are eligible to sit the JSCC cytotechnologist examination without 1 year of practical experience, but these schools are fewer than 10 and they remain a minor pathway. JSCC certified cytotechnologists provide both technical assistance and cytology screening services in the cytology laboratories of most institutions in Japan and often play a leading role in a central laboratory.

\section{PREPARATION AND STAINING OF THYROID CYTOLOGY SAMPLES}

A 22- or 23-gauge needle is often used instead of a 26- or 27gauge needle, in combination with a $10-\mathrm{mL}$ disposable syringe and a pistol-grip mechanical syringe holder. Local anesthesia is not provided. Two smear slides are usually prepared from one FNA specimen, and are fixed in 95\% ethanol or an equivalent fixative. Wet-fixed smears with Papanicolaou stain are favored by most laboratories, although some cytopathologists prefer airdried smears with Giemsa stain or Diff-Quik stain. Liquid-based cytology is used in some laboratories, but is not widely available. Examination of FNA needle washings (hormonal assays and other diagnostic markers) has recently become popular for confirmation of the cytological diagnosis.

\section{Thyroid FNA cytology reporting systems in Japan}

There are two sets of clinical guidelines for handling thyroid nodules. One is the General Rules for the Description of Thyroid Cancer (GRDTC) published by the Japanese Society of Thyroid Surgery (JSTS) in 2005 and updated in 2016. It includes a reporting system for thyroid FNA cytology. The other is a national reporting system (the Japanese system) for thyroid FNA cytology that was included in the clinical guidelines published by the Japan Thyroid Association (JTA) in 2013. ${ }^{8,9}$ The GRDTC reporting system was adapted from the Papanicoloau Society recommendations published in $1996,{ }^{10}$ while the Japanese system was adapted from Toriya's diagnostic system developed at Ito Hospital in Japan, which is characterized by two or three subclassifications of the indeterminate category. ${ }^{89}$ The Japanese system initially classifies the lesions in indeterminate category into indeterminate $\mathrm{A}$ (follicular neoplasms without PTC-type nuclear features [PTC-N]) and indeterminate $\mathrm{B}$ (nodules with nuclear atypia). The indeterminate $A$ is further classified into three subcategories: A1 (favor benign), A2 (borderline), and A3 (favor malignant) (Table 1). ${ }^{9}$ Although the Bethesda System for Reporting Thyroid Cytopathology (TBSRTC) itself is rarely used in Japan, the GRDTC and JTA system tried to incorporate TBSRTC so that cytological diagnoses made with the GRDTC or Japanese system could be related to TBSRTC diagnoses, and even the JSTS recommended TBSRTC after significant modifications. The GRDTC system is widely used in Japan, but several high-volume thyroid surgery centers prefer the Japanese system because they want to stratify the ROM in patients with FN. The indication for surgery is judged comprehensively by risk assessment on the basis of cytological diagnosis, ultrasound findings, and clinical findings. The prototype of the 
Japanese system has been used to reduce unnecessary diagnostic thyroidectomy in patients with FN cytology at several thyroid centers, including Ito Hospital (Tokyo, Japan) and Kuma Hospital (Kobe, Japan). As a result, it is not mandatory for Japanese patients with FN to undergo diagnostic thyroidectomy, which is completely different from the management in Western countries.

\section{Thyroid cytology classification according to the Japanese system}

In the Japanese system, thyroid nodules are classified into six

Table 1. Cytological reporting system recommended in the 2013 Japanese guideline for management of thyroid nodules

\begin{tabular}{lc}
\hline Diagnostic category & Risk of malignancy (\%) \\
\hline Inadequate (non-diagnostic) & 10 \\
Normal or benign & $<1$ \\
Indeterminate & \\
$\quad$ Indeterminate A (foliicular neoplasm) & \\
A-1: favor benign & $<15$ \\
A-2: borderline & $15-30$ \\
A-3: favor malignant & $40-60$ \\
Indeterminate B & $40-60$ \\
$\quad$ (others: atypia in non-follicular pattern lesions) & \\
Suspicious for malignancy & $>80$ \\
(not conclusive for malignancy) & $>99$ \\
Malignancy &
\end{tabular}

categories by cytological diagnosis (inadequate, benign, indeterminate $\mathrm{A}$, indeterminate $\mathrm{B}$, suspicious for malignancy, and malignant) (Table 1, Fig. 1). Indeterminate $A$ is a category for FN (equivalent to Bethesda IV, but excluding cases with PTC-N), which is divided into three subcategories (A1, A2, and A3) based on cytological findings and ROM. These cytological subcategories have an impact on making decisions about clinical management. The cytological features of indeterminate $\mathrm{A}$ are no colloid background, microfollicular growth, enlarged nuclei, 3-dimensional clusters, and high cellularity. Nodules that demonstrate prominent trabecular clusters, cellular atypia, loss of cellular polarity, loss of cohesiveness, and nuclear overlapping are classified as group A3 (Fig. 2), while those with sheet-like follicles, medium-sized follicles, and a low nucleus/cytoplasm ratio are classified as group A1 (Fig. 3). Group A2 is intermediate between groups $A 1$ and $A 3$. Oxyphilic follicular tumors are handled as a separate group from indeterminate $A$ and do not undergo subclassification in the Japanese system. Indeterminate B consists of lesions with nuclear abnormalities that are difficult to distinguish between benign and malignant, and are differentiated from FN by the presence of worrisome PTC-N. Lesions with extremely equivocal findings of PTC or medullary thyroid carcinoma (MTC) may be classified into this category. Indeterminate

The Japanese system and the Bethesda system

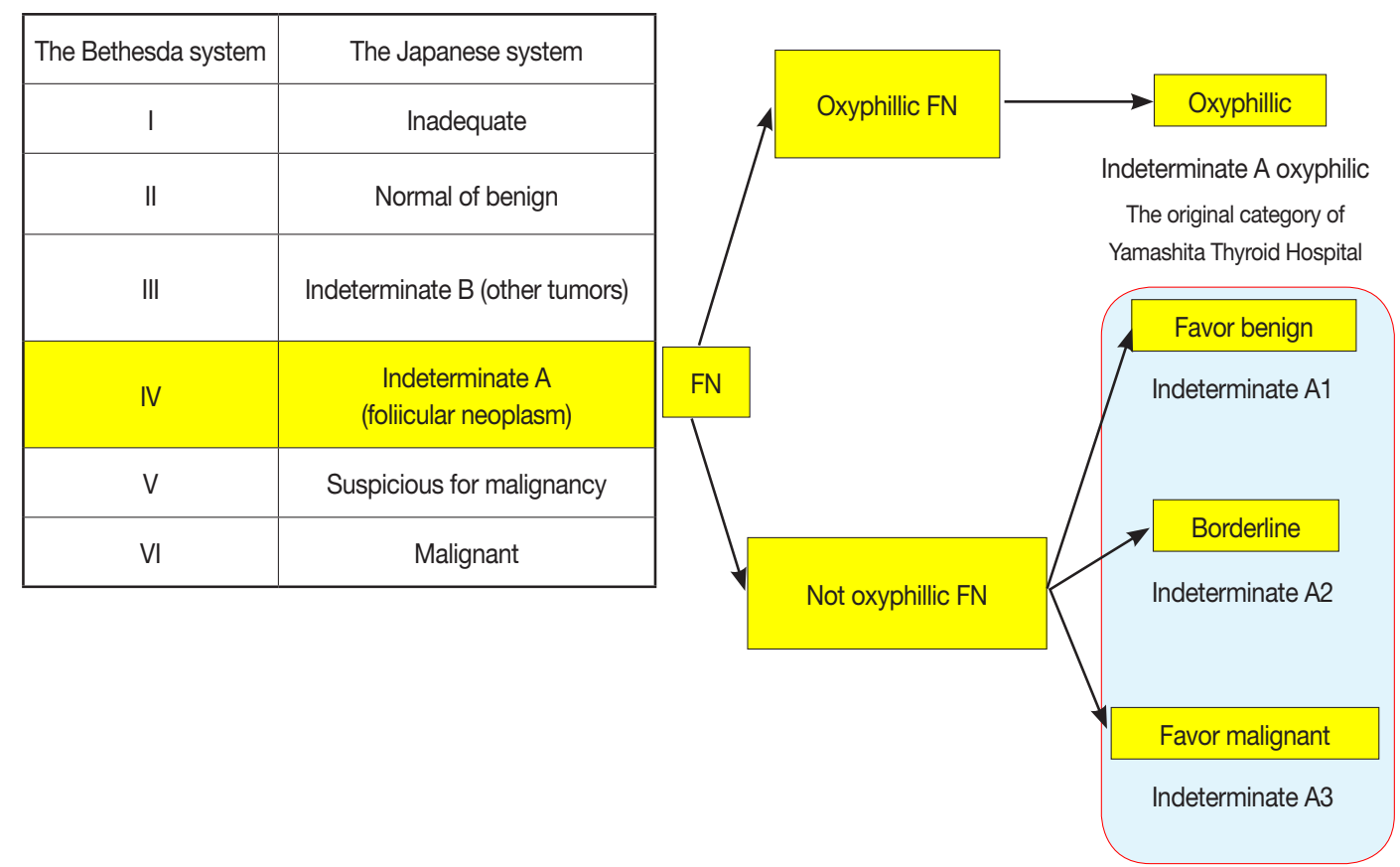

Fig. 1. Correlation between the Japan Thyroid Association reporting system (the Japanese system) and the Bethesda System for Reporting Thyroid Cytopathology (TBSRTC). FN, follicular neoplasm. 
B is almost equivalent to the Bethesda III (AUS/FLUS) category, but FLUS without PTC-N are excluded and classified as indeterminate A1 by the Japanese system. The other categories of the Japanese system (inadequate, benign, suspicious for malignancy, and malignant) are equivalent to those of TBSRTC (Fig. 1). A further modification of the Japanese system is classifying samples that only contain cyst fluid as benign because of an extremely low ROM. ${ }^{11}$

\section{The Japanese system in clinical practice at Yamashita Thyroid Hospital}

There are differences in surgical indications between Western and Asian practice, and the indications vary even among the institutions in Japan.

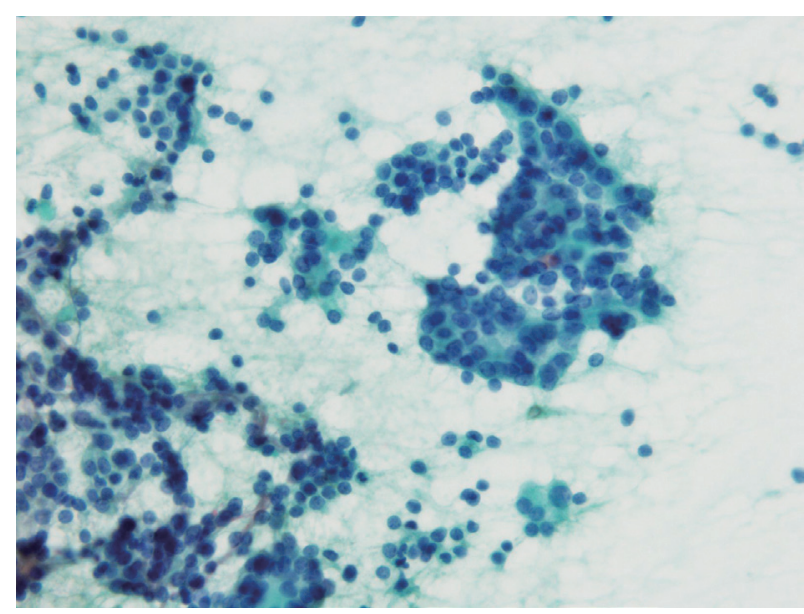

Fig. 2. Cytological findings of indeterminate A3. Cellular atypia, loss of cellular polarity, nuclear enlargement, and nuclear over-lapping are noted. The specimen was aspirated from a minimally invasive follicular carcinoma.

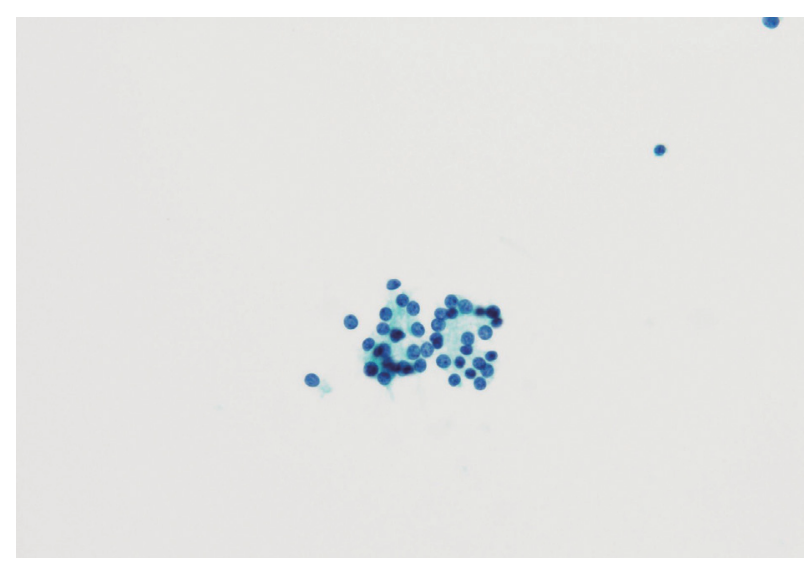

Fig. 3. Cytological findings of indeterminate A1. Microfollicular clusters are seen. The nuclei are round and slightly small. The specimen was aspirated from a follicular adenoma.
At Yamashita Thyroid Hospital, the indications for surgery in patients with thyroid nodules are as follows. Patients with inadequate cytology are recommended to undergo repeat FNA cytology or follow-up examination at intervals of 6 to 12 months. US is routinely performed at each follow-up visit in these patients. If a follicular tumor or cancer is suspected by US during the follow-up, repeat FNA cytology is usually performed. Patients with benign cytology are usually recommended to have follow-up examination, but diagnostic thyroidectomy may be recommended when the tumor is larger than $4 \mathrm{~cm}$ in diameter. Patients with indeterminate A1 cytology receive either diagnostic thyroidectomy or observation based on US findings and tumor size. Patients with indeterminate $\mathrm{A} 2$ or $\mathrm{A} 3$ cytology are recommended to undergo diagnostic thyroidectomy unless the tumor is small (e.g. $<1 \mathrm{~cm}$ in diameter). Although repeat FNA is recommended for patients with indeterminate $\mathrm{B}$ cytology, most patients with suspected malignancy according to US findings immediately undergo diagnostic thyroidectomy without repeat FNA. Patients with suspicious for malignancy or malignant cytology are recommended to undergo therapeutic thyroidectomy, but some patients with papillary microcarcinoma may be managed by observation, a so-called active surveillance. ${ }^{12-14}$

At Yamashita Thyroid Hospital (Fukuoka, Japan), about 1,000 surgical procedures for thyroid disease are carried out every year, and the results of US-guided FNA cytology for 1,600 nodules from January 2015 to April 2016 are summarized in Tables 2 and 3 . These tables show the correlation between cytological and histological diagnoses categorized according to either TBSRTC or the Japanese system. Because liquid-based cytology was not performed during this period, the frequency of Bethesda I (inadequate category) was high at $23.9 \%(382 / 1,600)$. The resection rate (RR), ROM in resected patients, and ROM in all patients receiving FNA are presented in Table 2. Among 154 nodules classified as Bethesda IV (Indeterminate A), 75 nodules were A1, 29 nodules were A2, and 11 nodules were A3. Thirtynine lesions were suspected to be oxyphilic follicular tumors and were separately classified as indeterminate A oxyphilic according to our original classification. The RR and ROM in each of these four subcategories are also listed in Table 3. We were able to successfully stratify category A into subcategories A1, A2, and A3 according to the increase of ROM and RR, thus reducing unnecessary thyroidectomies in patients with indeterminate $\mathrm{A}(\mathrm{FN})$.

The correlation between cytological and histological diagnoses in patients who underwent FNA cytology and surgical follow-up at Yamashita Thyroid Hospital is presented in Table 2. While PTC accounted for $98 \%$ of Bethesda VI, only two MTCs, one 


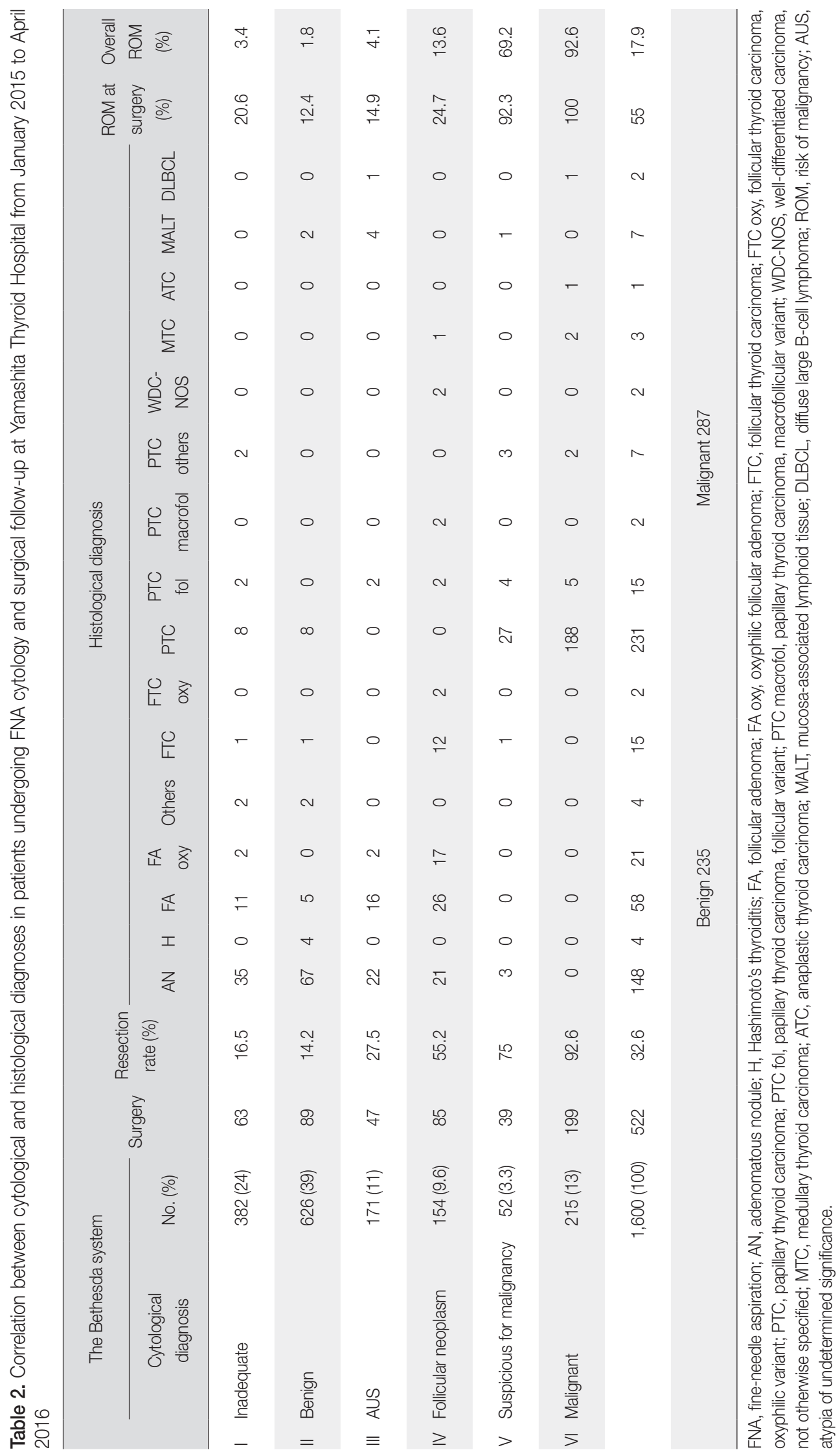


Table 3. Resection rate and risk of malignancy in patients with indeterminate A cytology (including oxyphilic follicular neoplasm)

\begin{tabular}{|c|c|c|c|c|c|c|c|c|c|c|c|}
\hline \multirow[b]{2}{*}{ Cytological classification } & \multicolumn{2}{|c|}{ №. (\%) } & \multirow[b]{2}{*}{ Malignancy } & \multirow{2}{*}{$\begin{array}{c}\text { ROM at } \\
\text { histology (\%) }\end{array}$} & \multirow{2}{*}{$\begin{array}{c}\text { Overall } \\
\text { ROM (\%) }\end{array}$} & \multicolumn{6}{|c|}{ Histological classification (malignancy) } \\
\hline & FNA-cytology & Resection & & & & FTC & $\begin{array}{l}\text { FTC } \\
\text { oxy }\end{array}$ & $\begin{array}{c}\text { PTC } \\
\text { fol }\end{array}$ & PTC macrofol & $\begin{array}{l}\text { WDC- } \\
\text { NOS }\end{array}$ & MTC \\
\hline Indeterminate A1 & $75(48.7)$ & $33(44)$ & 4 & 12.1 & 5.3 & 2 & 1 & - & 1 & - & - \\
\hline Indeterminate A2 & $29(18.8)$ & $20(68.9)$ & 10 & 50 & 34.5 & 7 & - & - & 1 & 2 & - \\
\hline Indeterminate A3 & $11(7.1)$ & $10(90.9)$ & 6 & 60 & 54.5 & 3 & - & 2 & - & - & 1 \\
\hline \multirow[t]{2}{*}{ Indeterminate A oxyphilic } & $39(25.3)$ & $22(56.4)$ & 1 & 4.5 & 2.6 & - & 1 & - & - & - & - \\
\hline & $154(100)$ & $85(55.2)$ & 21 & 24.7 & 13.6 & 12 & 2 & 2 & 2 & 2 & 1 \\
\hline
\end{tabular}

FNA, fine-needle aspiration; ROM, risk of malignancy; FTC, follicular thyroid carcinoma; FTC oxy, follicular thyroid carcinoma, oxyphilic variant; PTC fol, papillary thyroid carcinoma, follicular variant; PTC macrofol, papillary thyroid carcinoma, macrofollicular variant; WDC-NOS, well-differentiated carcinoma, not otherwise specified; MTC, medullary thyroid carcinoma.

anaplastic thyroid carcinoma and one diffuse large B-cell lymphoma were included in this category. Similarly, PTC accounted for more than $90 \%$ of the malignant tumors in Bethesda V. FTC accounted for about $70 \%$ of the malignant tumors in Bethesda IV (indeterminate A), while follicular variant PTC accounted for $20 \%$. In each indeterminate A subcategory (A1, A2, and $\mathrm{A} 3$ ), more than half of the malignant tumors had a histological diagnosis of FTC. In our hospital, the final histological diagnosis was FTC for the majority of malignancies in indeterminate A.

In 2015, Sugino et al. ${ }^{15}$ reported on the correlation between cytological and histological diagnosis in indeterminate A patients according to the Japanese system. They assessed 1,553 indeterminate A patients (4.3\%) among 36,066 patients who underwent US-guided FNA thyroid cytology between 2005 and 2011 at Ito Hospital (Tokyo, Japan). Histological diagnosis of the primary thyroid tumor was available in 779 of the 1,553 patients with indeterminate A lesions. The overall RR was $50.2 \%$, with the RR being $46.9 \%$ in A1, $55.4 \%$ in A2, and $65.1 \%$ in A3. The overall ROM was $34.5 \%$ in resected cases, while the ROM was $30.0 \%$ in resected $\mathrm{A} 1$ cases, $40.3 \%$ in $\mathrm{A} 2$, and $50.0 \%$ in $\mathrm{A} 3$. In addition, FTC accounted for about $75.5 \%$ of malignant tumors in indeterminate A patients, and 11 follicular variant PTCs (4.1\%) were included in indeterminate A category. Their results are fairly consistent with our findings.

In conclusion, the Japanese system seems to direct high-risk patients with FN to surgery, while low-risk patients with FN are recommended for follow-up. FTC accounted for the majority of malignancies in Japanese patients with indeterminate A lesions who underwent diagnostic thyroidectomy, which is different from reports on Western patients. ${ }^{16,17}$

\section{ANCILLARY TESTING, INCLUDING CORE BIOPSY}

Core needle biopsy is rarely performed in thyroid patients in
Japan; it may be used instead of open biopsy to confirm malignant lymphoma of the thyroid or undifferentiated carcinoma in inoperable cases. Molecular testing for BRAF mutation and other molecular alterations, as suggested in the 2015 guidelines of the American Thyroid Association, ${ }^{18}$ may be used to triage patients with indeterminate nodules, but is not a common practice in Japan. As of 2017, it is only done for academic research ${ }^{19}$ because its use is not supported by the Japanese national health insurance system. On the other hand, measurement of thyroglobulin ( $\mathrm{Tg}$ ) and/or calcitonin in FNA needle washings is often used as a helpful diagnostic adjunct in patients with thyroid nodules or cervical tumors, as are serum tumor markers. When the needle aspirate is immediately fixed and submitted for cytological examination, the needle and syringe are washed with $0.5 \mathrm{~mL}$ of saline and the washings are also submitted for measurement of biochemical markers. The presence of Tg in the FNA needle washings suggests that the tumor originated from thyroid follicular cells. This method is useful for establishing the diagnosis of lymph node metastasis when samples are obtained from possible cervical metastases of well-differentiated thyroid carcinoma. In 1983, Miyauchi et $a l^{20}$ (one of the leaders in the field of thyroid oncology in Japan and Asia) reported that the detection of very high thyroglobulin content in aspirates from cystic lymph nodes of the neck supported the diagnosis of metastatic PTC. This method is also applied to the diagnosis of MTC. Kudo (a colleague of Miyauchi) et $a l^{21}$ reported that the measurement of calcitonin in FNA needle washings could identify MTC with a high sensitivity and specificity. This is the historical background that explains why the measurement of $\mathrm{Tg}$ and/or calcitonin in aspirates is preferred by endocrinologists and endocrine surgeons in Japan.

\section{CONCLUSION}

In Japan, the clinician (endocrinologist, endocrine surgeon, or head and neck surgeon) usually performs FNA cytology at an 
outpatient clinic, while it is rarely done by pathologists, and US-guided FNA is common.

A 22- or 23-gauge needle is popular instead of a 26- or 27gauge needle. Wet-fixed smears (95\% ethanol) and Papanicolaou stain are favored by most laboratories in Japan.

The most popular reporting system for thyroid FNA cytology in Japan is the GRDTC adapted from the 1996 Papanicolaou Society recommendations. Although TBSRTC was introduced to replace the GRDTC, it has not yet become popular in Japan. The Japanese system is used at several high-volume thyroid surgery centers to stratify the ROM for FN nodules.

Cytological diagnoses made by the GRDTC or Japanese system correspond to those of TBSRTC.

At high-volume thyroid centers, the Japanese system has been useful for risk stratification in patients with FN nodules and it has reduced unnecessary diagnostic thyroidectomy.

The measurement of $\mathrm{Tg}$ and/or calcitonin in FNA needle washings is often used as a diagnostic adjunct in patients with possible cervical lymph node metastasis.

\section{Conflicts of Interest}

No potential conflict of interest relevant to this article was reported.

\section{REFERENCES}

1. Soderstrom N. Puncture of goiters for aspiration biopsy. Acta Med Scand 1952; 144: 237-44.

2. Okui K, Lindberg LG. Aspiration cytology in Lund University Hospital, Sweden. J Jpn Soc Clin Cytol 1979; 18: 105-9.

3. Toriya K. Cytological aspiration biopsy of goiters, especially with reference to their histological and cytological examinations. J Jpn Soc Clin Cytol 1972; 11: 101-14.

4. Toriya K. Fine needle aspiration cytology of the thyroid. Shindantochiryou 2005; 93: 1051-5.

5. Ali SZ, Cibas ES. The Bethesda system for reporting thyroid cytopathology: definitions, criteria and explanatory notes. New York: Springer, 2010.

6. Kakudo K, Kameyama K, Miyauchi A. History of thyroid cytology in Japan and reporting system recommended by the Japan Thyroid Association. J Basic Clin Med 2013; 2: 10-5.

7. Japanese Society of Thyroid Surgery Editorial Committee. General rules for the description of thyroid cancer. 6th ed. Tokyo: Kanahara Publisher, 2005.

8. Japan Thyroid Association. Guidelines for clinical practice for the management of thyroid nodules in Japan 2013. Tokyo: Nankodo Co., Ltd., 2013.

9. Kakudo K, Kameyama K, Miyauchi A, Nakamura H. Introducing the reporting system for thyroid fine-needle aspiration cytology according to the new guidelines of the Japan Thyroid Association. Endocr J 2014; 61: 539-52.

10. Guidelines of the Papanicolaou Society of Cytopathology for the examination of fine-needle aspiration specimens from thyroid nodules. The Papanicolaou Society of Cytopathology Task Force on Standards of Practice. Diagn Cytopathol 1996; 15: 84-9.

11. Takada N, Hirokawa M, Suzuki A, Higuchi M, Kuma S, Miyauchi A. Reappraisal of "cyst fluid only" on thyroid fine-needle aspiration cytology. Endocr J 2017; 64: 759-65.

12. Miyauchi A. Clinical trials of active surveillance of papillary microcarcinoma of the thyroid. World J Surg 2016; 40: 516-22.

13. Leboulleux S, Tuttle RM, Pacini F, Schlumberger M. Papillary thyroid microcarcinoma: time to shift from surgery to active surveillance? Lancet Diabetes Endocrinol 2016; 4: 933-42.

14. Brito JP, Ito Y, Miyauchi A, Tuttle RM. A clinical framework to facilitate risk stratification when considering an active surveillance alternative to immediate biopsy and surgery in papillary microcarcinoma. Thyroid 2016; 26: 144-9.

15. Sugino K, Kameyama K, Ito K. Characteristics and outcome of thyroid cancer patients with indeterminate cytology. J Basic Clin Med 2015; 4: 92-8.

16. Yang J, Schnadig V, Logrono R, Wasserman PG. Fine-needle aspiration of thyroid nodules: a study of 4703 patients with histologic and clinical correlations. Cancer 2007; 111: 306-15.

17. Williams BA, Bullock MJ, Trites JR, Taylor SM, Hart RD. Rates of thyroid malignancy by FNA diagnostic category. J Otolaryngol Head Neck Surg 2013; 42: 61.

18. Haugen BR, Alexander EK, Bible KC, et al. 2015 American Thyroid Association management guidelines for adult patients with thyroid nodules and differentiated thyroid cancer: the American Thyroid Association Guidelines Task Force on Thyroid Nodules and Differentiated Thyroid Cancer. Thyroid 2016; 26: 1-133.

19. Kumagai A, Namba H, Akanov Z, et al. Clinical implications of pre-operative rapid BRAF analysis for papillary thyroid cancer. Endocr J 2007; 54: 399-405.

20. Miyauchi A, Takai S, Morimoto S, et al. Fine needle aspiration of neck tumor. Application of cytological, bacteriological and hormonal examinations. Nihon Geka Gakkai Zasshi 1983; 84: 667-73.

21. Kudo T, Miyauchi A, Ito Y, Takamura Y, Amino N, Hirokawa M. Diagnosis of medullary thyroid carcinoma by calcitonin measurement in fine-needle aspiration biopsy specimens. Thyroid 2007; 17: 635-8. 